\title{
Telencephalon of the Teleost Macropodus: Experimental Localization of Secondary Olfactory Areas and of Components of the Lateral Forebrain Bundle
}

\author{
Roger E. Davis, Robin Chase, Joseph Morris, and Bernice Kaufman ${ }^{1}$ \\ Mental Health Research Institute and Neuroscience Laboratory, University of Michigan, \\ Ann Arbor, Michigan 48109
}

\begin{abstract}
Connections of the telencephalic hemisphere were experimentally examined to facilitate investigations of the functions of this major brain area in teleosts. The distribution of afferent olfactory tract fibers was traced using a degeneration method and autoradiographic localization of axonally transported protein. Afferents terminate predominantly ipsilaterally in the medial, lateral, and posterior zones of the hemisphere and in the nucleus posterior tuberis of the diencephalon. Afferents also project to the contralateral olfactory bulb through the commissure of Goldstein. The results of similar experiments in five other teleosts are briefly reviewed. Evidence of retrograde transport of tritiated proline or metabolite is also discussed. Components of the lateral forebrain bundle originating in the extreme rostral end of the dorsal zone of the hemisphere were traced to: (1) the corresponding zone of the contralateral hemisphere; (2) nucleus entopenduncularis; and (3) the extreme ventral part of the posterior zone of the diencephalon. The anatomical findings are discussed in relation to previous experiments in Macropodus dealing with the location of brain areas which concentrate sex steroids and with the effects of brain lesions on reproductive behavior in the male.
\end{abstract}

Telencephalon ablation experiments in the paradise fish, Macropodus opercularis, indicate that the hemisphere mediates processes which potentiate male reproductive behaviors (Davis, Kassel, \& Schwagmeyer, 1976; Kassel, Davis \& Schwagmeyer, 1976; Kassel \& Davis, 1977; Schwagmeyer, Davis, \& Kassel, 1977). Bilateral ablation of the hemispheres and olfactory bulbs results in a decreased incidence of mating with intact females and increased egg cannibalism. Removal of only the bulbs results in increased egg eating, but sexual behavior is unimpaired. The results indicate olfactory areas in the brain are necessary for normal egg care and that nonolfactory structures are sufficient for the potentiation of sexual behavior (Davis, Kassel, \& Martinez, 1981).

'We thank R. Glenn Northcutt and Catherine McCormick for assistance in the degeneration staining procedure. Requests for reprints should be sent to R. E. Davis. 
The olfactory functions of the hemisphere in teleosts have received little direct attention in behavioral studies. Brain lesion and electrical stimulation experiments have concentrated on nonolfactory processes such as reinforcement (Flood, Overmier, \& Savage, 1976), arousal (Laming, 1980, 1981), potentiation of sexual behavior (Davis et al., 1981; Demski \& Knigge, 1971; Demski, Bauer, \& Gerald, 1975), and startle reactivity (Davis, Reynolds, \& Ricks, 1978). The nature of these processes remains ill-defined, and attempts to localize the structures responsible have generally produced ambiguous results. We believe that the olfactory functions of the hemisphere may be more amenable to investigation. As a foundation for such research, the cytoarchitecture of the telencephalon of Macropodus was described, and the afferent projections of the olfactory tracts were investigated using a degeneration method and autoradiographic localization of axonally transported protein.

\section{METHOD}

\section{Cytoarchitecture and Terminology}

The major brain-cell groups, or nuclei, were identified in transverse and horizontal sections of reference brains, which were stained either by the Bodian method, by the Kluver-Barrera method, or with cresylviolet acetate. The identifications were based on descriptions of the brain of Gasterosteus (Nieuwenhuys, 1962), Carassius (Peter \& Gill, 1975), and Lepomis (Northcutt \& Braford, 1980). The terminology of Nieuwenhuys (1962,1963), as modified by Northcutt and Braford (1980), was adopted (see Table 1).

\section{Autoradiographic Method}

Adult male Macropodus opercularis (L.), 4.7-5.5 cm body length, were obtained from a local supplier. Fish each received $0.2-0.5 \mu \mathrm{Ci}$ of $\mathrm{L}-\left[2,3-{ }^{3} \mathrm{H}\right]$ proline which was air-dried on a $60-80-\mu \mathrm{m}$ Dowex resin bead (Davis and Agranoff, 1977). The bead was implanted unilaterally in the olfactory bulb. In additional fish, the contralateral bulb was immediately removed to prevent transport of radiolabeled protein in the contralateral olfactory tracts. The results are based on four fish for which the autoradiograms revealed that uptake of radiolabeled proline was confined mainly to the olfactory bulb and peduncle and on four other fish that showed extensive uptake of proline in the contralateral bulb and the rostral pole of the ipsilateral hemisphere.

Fish were sacrificed by brief immersion in ice slush 10 days following the proline administration. The brain was fixed in $10 \%$ formalin or alcohol-formalin-acetic acid, removed from the cranium, and embedded in paraffin. Serial, transverse, or horizontal sections of the brain, cut 10 $\mu \mathrm{m}$ thick, were mounted on glass slides for autoradiography (Kopriwa 
TABLE 1

Abbreviations Used in Figs. 1-10

\begin{tabular}{|c|c|}
\hline Abbreviation & Meaning \\
\hline $\mathrm{AC}$ & Anterior commissure \\
\hline CG & Commissure of Goldstein \\
\hline D & Area Dorsalis telencephali \\
\hline $\mathrm{Db}$ & Dowex resin bead \\
\hline Dc & Central part of $\mathrm{D}$ \\
\hline Dc- $1,2,3,4$ & Divisions of Dc \\
\hline Dd & Dorsal part of $\mathrm{D}$ \\
\hline DI & Lateral part of $\mathrm{D}$ \\
\hline Dld,p,v & Dorsal, posterior, and ventral divisions of $\mathrm{Dl}$ \\
\hline $\mathrm{Dm}$ & Medial part of D \\
\hline Dm-1,2,3,4 & Divisions of Dm \\
\hline DMC & Commissure of the medial part of $D$ \\
\hline Dp & Posterior part of D \\
\hline Ed,v & Dorsal and ventral divisions of nucleus entopeduncularis \\
\hline GL & Glomerular layer \\
\hline ICL & Internal cell layer \\
\hline it & Lateral olfactory tract \\
\hline LFB & Lateral forebrain bundle \\
\hline M & Meningeal tissue \\
\hline MO & Medulla oblongata \\
\hline $\mathrm{mc}$ & Caudal branch of $\mathrm{mt}$ \\
\hline md & Dorsal branch of $\mathrm{mt}$ \\
\hline $\mathrm{mm}$ & Medial branch of $\mathrm{mt}$ \\
\hline $\mathrm{mt}$ & Medial olfactory tract \\
\hline $\mathbf{N}$ & Nervus terminalis \\
\hline NAT & Nucleus anterior tuberis \\
\hline NDLI & Nucleus diffusus lobi inferioris \\
\hline NDTL & Nucleus diffuses tori lateralis \\
\hline NG & Nucleus glomerulosus \\
\hline $\mathrm{NH}$ & Nucleus habenularis \\
\hline NLTi & Nucleus lateral tuberis parts inferioris \\
\hline NPG & Nucleus preglomerulosus \\
\hline NPT & Nucleus posterior tuberis \\
\hline NSV & Nucleus saccus vasculosus \\
\hline NT & Nucleus taenia \\
\hline $\mathrm{Ob}$ & Olfactory bulb \\
\hline $\mathrm{T}$ & Telencephalon \\
\hline ON & Olfactory nerve layer \\
\hline Pit & Pituitary \\
\hline POA & Preoptic nuclei \\
\hline PR & Preoptic recess \\
\hline SOF & Secondary olfactory fiber layer \\
\hline Otec & Optic tectum \\
\hline $\mathrm{V}$ & Area Ventralis telencephali \\
\hline $\mathrm{Vc}, \mathrm{d}, \mathrm{i}, 1, \mathrm{p}, \mathrm{s}$ & $\begin{array}{l}\text { Central, dorsal, intermediate lateral, postcommissural, and } \\
\text { supracommissural parts of } \mathrm{V}\end{array}$ \\
\hline $\mathrm{X}$ & Nucleus $\mathrm{X}$ \\
\hline
\end{tabular}


\& Leblond, 1962; Landreth \& Agranoff, 1976). The slides were dipped in Kodak NTB-3 emulsion, dried, and stored at $4^{\circ} \mathrm{C}$ in darkness for 21 days. The emulsion was developed in Kodak Dektol for $3 \mathrm{~min}$ at $16^{\circ} \mathrm{C}$, washed, and fixed. The sections were lightly stained with cresyl-violet acetate. The distribution of grains of reduced silver in the emulsion over the sections was examined by bright and dark field microscopy.

\section{Degeneration Method}

The olfactory bulb was unilaterally aspirated in 14 fish. Following a survival time of 2 to 10 days, the fish was sacrificed, the roof of the cranium removed, and the head fixed in $10 \%$ formalin for a minimum of 1 week. In preparation for the silver impregnation procedure (Wiitanen, 1969), the fixed brain was removed, washed overnight, and infiltrated and embedded in $20 \%$ gelatin. The gelatin block was subsequently fixed in $25 \%$ formalin overnight and transferred to $10 \%$ formalin for storage prior to cutting. The block was frozen on the stage of a sliding microtome following overnight immersion in 1:1 10\% ethanol and 10\% formalin. Frozen transverse or horizontal $35-\mu \mathrm{m}$ sections were cut and stored in $2 \%$ formalin at $4^{\circ} \mathrm{C}$ until the impregnation process was carried out.

Degenerating fibers were identified by the occurrence of localized deposits of grains of reduced silver in the tissue, which could be traced in successive sections from the lesioned olfactory bulb. The results are based on four fish that survived $2,4,8$ or 10 days. In these fish, the lesion included the bulb and part of the olfactory peduncle and the contralateral bulb was not visibly damaged.

\section{RESULTS \\ I. Cytoarchitecture}

\section{A. Olfactory Bulb}

The bulb is sessile, or closely apposed to the hemisphere, as in most other teleosts (Nieuwenhuys, 1967). The short olfactory peduncle, which contains the olfactory tracts, is overhung by the rostral pole of the hemisphere. The olfactory nerve layer and the underlying glomerular layer surround the bulb rostrally (Fig. 1A). In the caudal region, these layers are restricted to the ventral and lateral quadrants. In addition to the primary afferent fibers, the nerve also contains fibers that terminate in the receptor epithelium as described elsewhere (Davis, 1979). The mitral cells and secondary olfactory fibers are intermingled and do not form distinct layers. Scattered, large perikarya, presumed to be mitral cells, also occur in the glomerular layers (Holmgren, 1920). The internal cell layer, which may be homologous with the anterior olfactory nucleus 


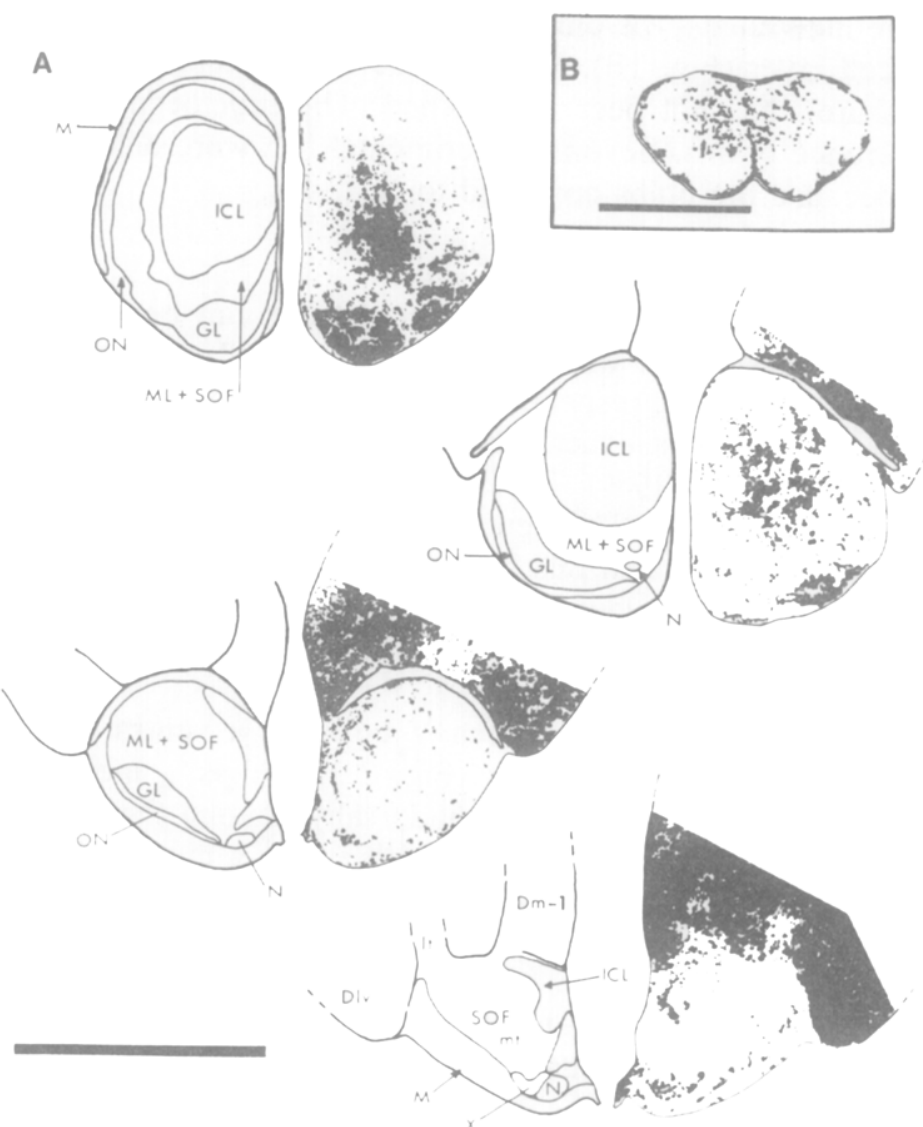

Fig. 1. (A) Transverse sections of the olfactory bulbs. The most rostral section (top) is from the middle of the bulb. Kluver-Barrera stain. (B) Example of fused olfactory bulbs. Cresyl-violet acetate stain. Scale bars $=0.5 \mathrm{~mm}$. Abbreviations are presented in Table 1.

of land vertebrates (Northcutt \& Braford, 1980), tapers caudomedially into the peduncle.

In the peduncle, the mass of secondary olfactory fibers separates into the lateral and medial divisions of the olfactory tracts. The lateral tract enters the hemisphere dorsolaterally and the medial tract ventromedially. Large perikarya, designated as nervus terminalis ganglion cells $(\mathrm{N})$, are distributed rostrocaudally in the ventromedial zone of the bulb and peduncle. As shown in Fig. 1A, in the peduncle the $\mathrm{N}$ cells occur in close association with a small cluster of very large perikarya, which are identified as nucleus " $X$ " (Ariens-Kappers, Huber, \& Crosby, 1936). This nucleus may be a part of the nervus terminalis system (Northcutt \& Braford, 1980). 
In some individuals, the olfactory bulbs are fused, forming a common internal cell layer (Fig. 1B). Whether the bulbs exchange fibers in this "commissure" has not been determined. The trait occurred in one of eight reference brains. Several experimental fish were discarded during surgery because the bulbs appeared to be fused.

\section{B. Hemisphere}

The hemisphere of the teleost telencephalon can be divided into a dorsal and ventral zone, which are thought to be homologous with the pallium and subpallium, respectively, of land vertebrates (Nieuwenhuys, $1962,1963)$, although the exact boundary between the two is controversial (Northcutt \& Braford, 1980). The dorsal zone is denoted as area Dorsalis telencephali, or area D. The ventral zone is denoted as area Ventralis telencephali, or area V. In this report, area V is deemed to include the caudoventral region between the anterior commissure and the habenular nucleus of the thalamus. We identified 23 nuclei, or brain-cell groups, as illustrated in Fig. 2.

Area $V$. The rostral part of area $\mathrm{V}$ contains a ventral $(\mathrm{Vv})$, dorsal (Vd), and a lateral (V1) nucleus (Fig. 2.3). Vd is a morphologically complex group, and it extends caudodorsally beyond the anterior commissure (Fig. 2.6). Vv is subjacent to $\mathrm{Vd}$ and is a more circumscribed, uniform nucleus. $\mathrm{Vv}$ tapers caudodorsally over the preoptic recess, and it is replaced at the anterior commissure by the supracommissural nucleus (Vs; Fig. 2.4). V1 consists of an irregular strand of cells in the lateral wall of $\mathrm{V}$ (Fig. 2.3). The cells are larger and more dispersed than the cells of $\mathrm{Vv}$ or $\mathrm{Vd}$. Widely scattered cells occur in the central part of $\mathrm{V}$. While they may belong to the central nucleus of $\mathrm{V}$, as seen in Lepomis (Northcutt \& Braford, 1980), a distinct grouping of these cells was not evident in our material.

Posteriorly (Fig. 2.5-2.7), the medial wall of $\mathrm{V}$ contains the caudal part of $\mathrm{Vd}$, the postcommissural nucleus $(\mathrm{Vp})$, and the preoptic region (POA). More laterally, a ventral (Ev) and a dorsal (Ed) entopeduncular nucleus can be distinguished. Ev is a highly compact, elongated group. Ed, a smaller, more dispersed group, extends caudally to the intermediate nucleus (Vi).

Area $D$. Area $\mathrm{D}$ consists of a peripheral zone containing nine relatively compact cell masses and a central zone of larger cells, which are dispersed singly and in several small groups. The peripheral groups appear to be subdivisions of three rostrocaudal columns of brain cells, a dorsomedial (Dm), dorsal (Dd), and a dorsolateral (D) column (Northcutt \& Braford, 1980). The dorsal column consists of a unitary group (Dd; Fig. 2.1), which tapers caudally and disappears. Column Dm has three parts. The most rostral nucleus, Dm-1 (Fig. 2.2), is the smallest and it is replaced caudally by Dm-3 (Fig. 2.4). The ventral extreme of Dm-1 


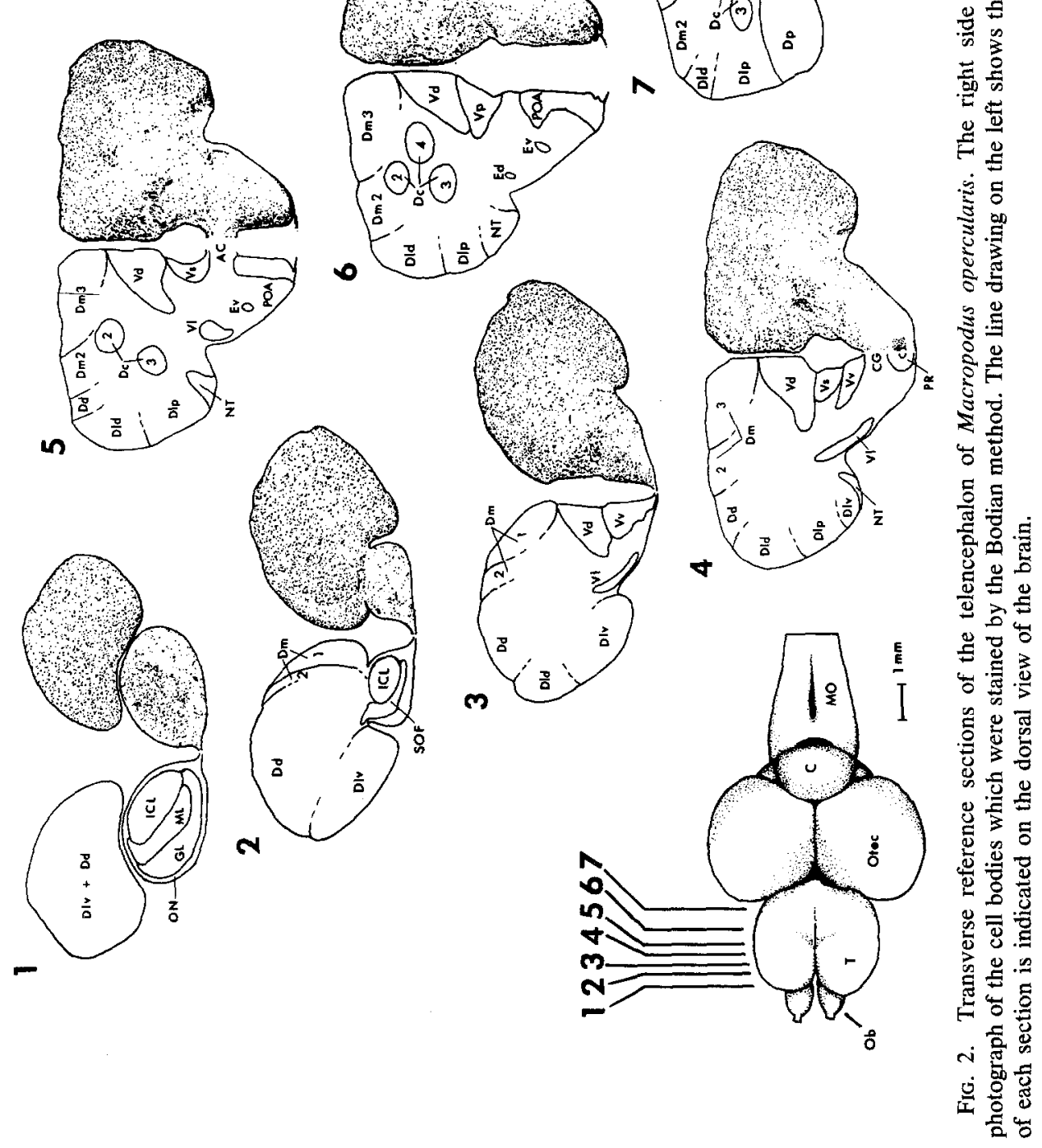


is apposed to the caudal portion of the olfactory bulb (Fig. 2.2). Dm-2 and 3 expand caudally and, in most individuals, Dm-3 coalesces, forming the commissure Dorsalis telencephali (DMC; Fig. 2.7). DMC has been detected in only a few teleosts, but it is typical in Macropodus and other members of the suborder Anabantoidei.

Column Dl includes four groups in the lateral wall of $D$ and the posterior nucleus (Dp). The ventral nucleus of $\mathrm{Dl}(\mathrm{Dlv})$ and $\mathrm{Dd}$ form the extreme rostral end of the hemisphere (Fig. 2.1). Dlv tapers caudoventrally between the dorsal nucleus of Dl (Dld) and nucleus taenia (NT) and is replaced by the posterior nucleus of Dl (Dlp; Fig. 2.4). NT is a distinct, wedge-shaped group which reaches caudally beneath Dlp to Dp and Vi (Fig. 2.4-2.6).

Four cell masses can be distinguished in the central zone of area $D$. Dc-1 occurs in the rostral region adjacent to Dm-1. Dc-2 is closely associated with Dm-2 (Fig. 2.5). Dc-3 and Dc-4 are distinctive features of the posterior zone of $\mathrm{D}$ (Fig. 2.7).

\section{Problems of Method}

\section{A. Autoradiography}

The autoradiographic method produced the clearest labeling of the central projections of the olfactory bulb. We assume that most of the label seen in the autoradiograms resulted from emissions from radioactive protein. Very little of the tritiated proline should remain in the brain 10 days and, in any case, the histological procedure presumably removes most amino acids. In support of this assumption, Fig. 5.1 shows that, in an olfactory bulb containing a high concentration of radioactivity in the central zone, the surrounding olfactory nerve layer was comparatively lightly labeled. Since protein is synthesized in cell bodies, the nerve layer, which consists mainly of the receptor axons, should contain relatively little radioactivity. The boundary between the nerve layer and the glomerular layer is indistinct, and some of the label grains over the area denoted as "olfactory nerve layer" may in fact mark the location of labeled glomerular elements among the receptor axons. The nerve layer also contains scattered small cells, which have been variously described as subglomerular or periglomerular cells (Nieuwenhuys, 1967). Diffusion of tritiated proline into the nerve layer could have resulted in labeled protein in such cells. Some periglomerular cells have been identified as neurons, possibly efferents, which project in the olfactory nerve to the receptor epithelium (Davis, 1979, and in preparation).

The extensive labeling of the contralateral bulb and the rostral pole of the ipsilateral hemisphere that occurred in some brains was interpreted as being the result of local uptake of tritiated proline that diffused from the implantation site. While the following description of the olfactory 
bulb projections is based on brains in which the contralteral bulb was ablated or only lightly labeled, the cases of bilateral labeling aided in visualizing the pathways.

The intense labeling of the hemisphere presented the problem of dissociating olfactory fibers from labeled fibers arising from hemispheric nucleic. Mainly, the hemisphere adjacent to the experimental bulb was affected. The zone of diffusion invaded the extreme rostral parts of areas $\mathrm{V}$ and $\mathrm{D}$. Whether fibers arising from cells in these nuclei merge with components of the olfactory tracts cannot be determined from this experiment. However, heavily labeled components of the lateral forebrain bundle were detected and were easily distinguished from the afferent olfactory system, as described below.

Relatively dense, tractlike concentrations of silver grains that could be traced from the olfactory bulb through successive brain sections were inferred to indicate the pathways of the olfactory tract fibers. Diffuse patterns of grains that were continuous with the label over a pathway were assummed to mark the location of fiber terminations.

\section{B. Degeneration Method}

Degenerating olfactory tract fibers, marked by coarse-grain deposits, could be traced through the anterior zone of the hemisphere, but caudal to the anterior commissure their distribution became increasimgly indistinct (Fig. 3). Caudally, fine-grain deposits characteristic of fiber terminations were visible in various nuclei. In addition to confirming certain autoradiographic findings in the caudal half of the hemisphere, the degeneration results aided in identifying terminal fields in rostral area $\mathrm{V}$, which were difficult to distinguish in the autoradiographs of brains in

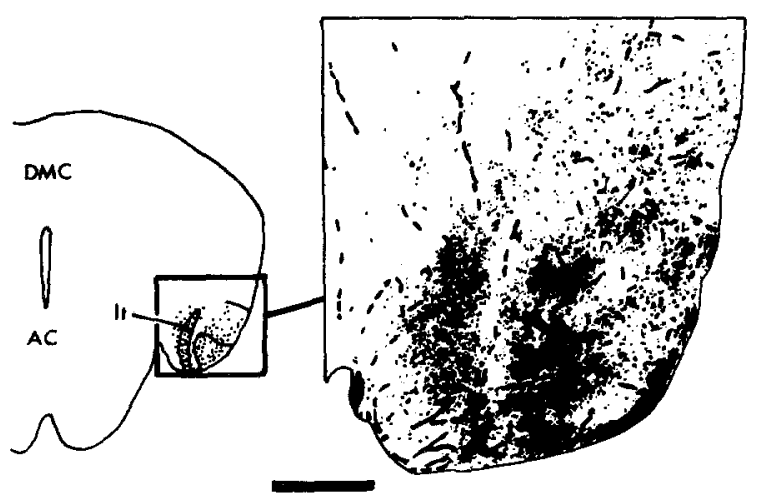

FIG. 3. Transverse section through the rostral end of nucleus taenia showing degenerating olfactory tract fibers. Fine-grain deposits indicative of degenerating terminals, which occurred in the area lateral to the core of the tract, are largely invisible in this photograph. Scale bar $=0.5 \mathrm{~mm}$. 
which the zone of diffusion of labeled proline from the experimental bulb was extensive.

\section{Distribution of Labeled Olfactory Tract Fibers}

The following description is accompanied by high-contrast, low-magnification photomicrographs of autoradiograms of selected horizontal or transverse brain sections that illustrate the most heavily labeled pathways. The distribution of labeled fibers is summarized in Figs. 9 and 10. Table 2 lists the nuclei that are inferred to receive olfactory system afferents and indicates the relative magnitude of the innervation which was inferred from the density of labeling.

\section{A. Ipsilateral Projections}

The medial and lateral divisions of the olfactory tract converge and intermingle in the caudal half of the hemisphere, and their separate pathways could not be traced with certainty. Thus, the tract-of-origin of the fiber terminations described below is uncertain. Upon entering the anterior part of $\mathrm{V}$, the medial olfactory tract forms a dorsal (md) and a medial (mm) branch of similar diameter (Figs. 4.3 and 6.5) and a smaller ventral branch corresponding to the interbulbar commissure of Goldstein described below (Figs. 5.4 and $8 \mathrm{~A}$ ). The dorsal and medial branches course caudally through diffuse terminal fields bordering nuclei $\mathrm{Vd}$ and $\mathrm{Vv}$. The anterior central part of the area $\mathrm{V}$ was also diffusely labeled, particularly in the autoradiograms (Fig. 4.3), suggesting that olfactory afferents terminate there. Degeneration data revealed a minute bundle entering the rostral extreme of area $\mathrm{V}$ ventral to the medial olfactory tract (not illustrated) corresponding to the nervus terminalis as described by Sheldon (1912). The degenerating fibers disappeared caudally near the preoptic recess.

The dorsal and medial branches of the medial tract are most readily visualized in horizontal sections (Fig. 7). The medial branch forms a commissural and a caudal (mc) branch (Figs. 4.7, 6.6, and 6.7). The commissural branch appears to merge with fibers of the lateral tract in

TABLE 2

Structures Inferred to Receive Secondary Olfactory Afferents

\begin{tabular}{llll}
\hline & \multicolumn{3}{c}{ Specific structure and degree of innervation } \\
\cline { 2 - 3 } Brain region & \multicolumn{1}{c}{ High } & Intermediate & Low \\
\hline Area V & $\mathrm{Vi}^{a}$ & $\mathrm{Vv}, \mathrm{Vd}$ & $\mathrm{Vs}^{a}{ }^{a} \mathrm{Vp}^{a},{ }^{a}$ POA \\
Area D & $\mathrm{NT},{ }^{a} \mathrm{Dlp},{ }^{a}{ }^{\mathrm{Dp}}{ }^{a}$ & $\mathrm{Dc}-3$ & $\mathrm{Dlv}$ \\
Diencephalon & $\mathrm{NPT}$ & \\
Contralateral olfactory bulb & Indeterminant & & \\
\hline
\end{tabular}

${ }^{a}$ Innervated bilaterally. 


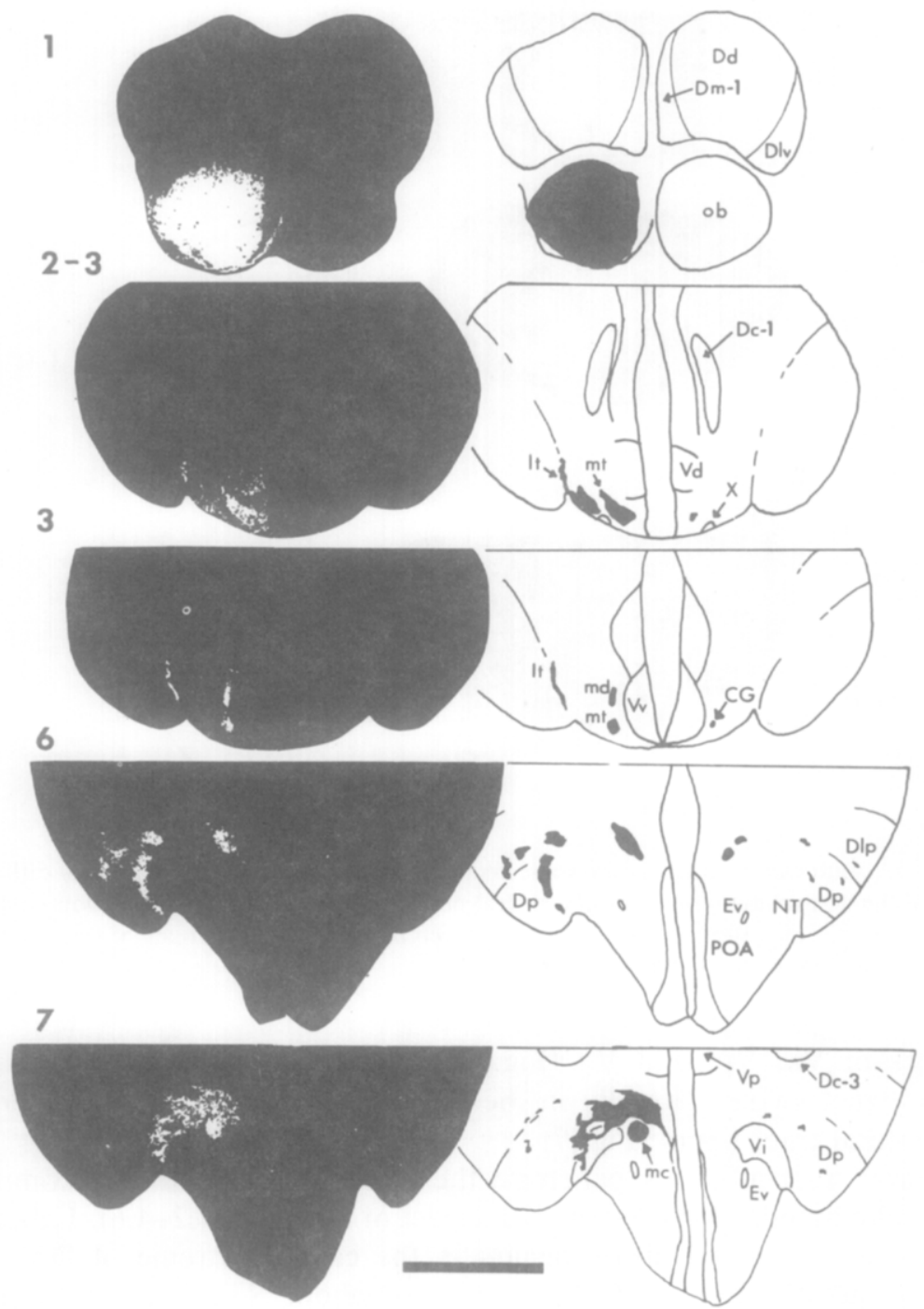

FiG. 4. Autoradiograms of transverse sections of a brain in which the zone of diffusion was largely restricted to the experimental bulb. The number to the left of each section refers to the closest reference section shown in Fig. 2. The locations of the main components of the radiolabeled tracts are illustrated on the line drawings. Scale bar $=0.5 \mathrm{~mm}$.

the dorsal region of the anterior commissure (Figs. 6.5, 7A). The caudal branch descends to the diencephalon and terminates in the region of nucleus posterior tuberis (NPT) (Fig. 10). The caudal branch may also be joined by lateral tract fibers as it courses through the postcommissural zone of the hemisphere. In this region, sparse terminal fields invade the 

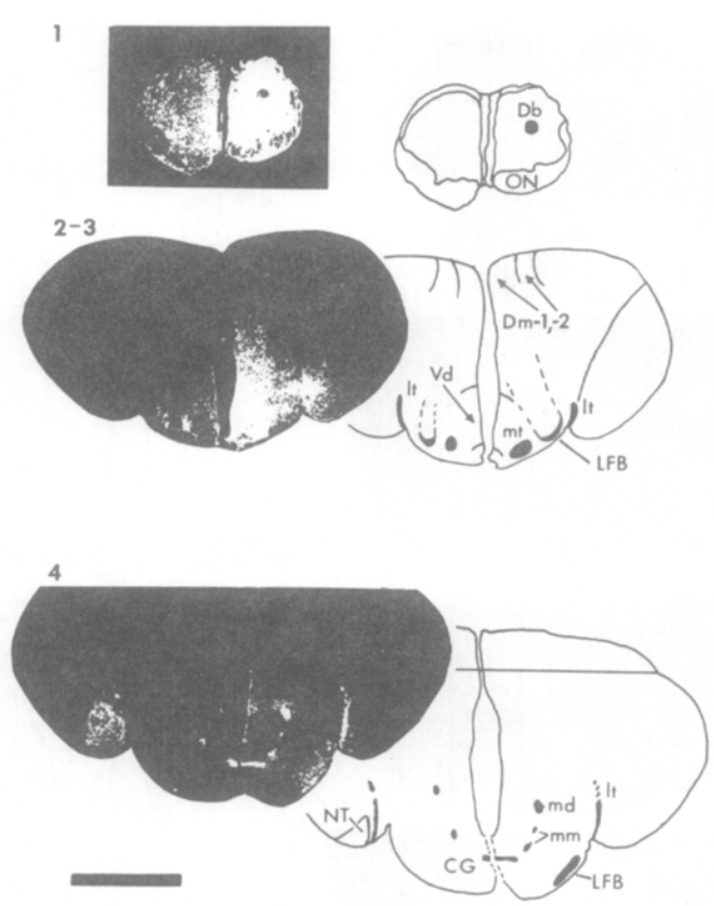

Fig. 5. Autoradiograms of transverse sections of a brain in which the zone of diffusion invaded the contralateral bulb and the rostral pole of the hemisphere. The section numbers indicate the closest reference section shown in Fig. 2. Additional sections of this brain are illustrated in Fig. 6. Scale bar $=0.5 \mathrm{~mm}$.

borders of Vs, Vp, and Vd (Fig. 9.4-9.6). The dorsal branch of the medial tract swings laterally in the post commissural zone and merges with lateral tract fibers (Fig. 7).

Lateral tract afferents penetrate the hemisphere, forming a compact ribbonlike bundle along the medial aspect of Dlv (Fig. 4.2-4.6). Labeling indicative of terminal fields occurs in the caudal extreme of Dlv, the ventral part of Dlp and of Dc-3, and throughout NT, Dp, and Vi (Fig. 10). Some or all of these areas could receive medial tract fibers (md) as well as lateral tract fibers. Based on the density of silver grains over the cells, nuclei Dlp, Dp, and Vi are the predominate targets of the olfactory afferents (Table 2). Some lateral tract fibers appear to swing medially and combine with medial tract fibers in the anterior commissure as described above. A small bundle of fibers descends from the region of $\mathrm{Vi}$ to the dorsal margin of POA (Figs. 4.7 and 6.6) and another projects from the caudal extreme of the hemisphere along the medial aspect of nucleus Ev into the habenular commissure (not illustrated). 


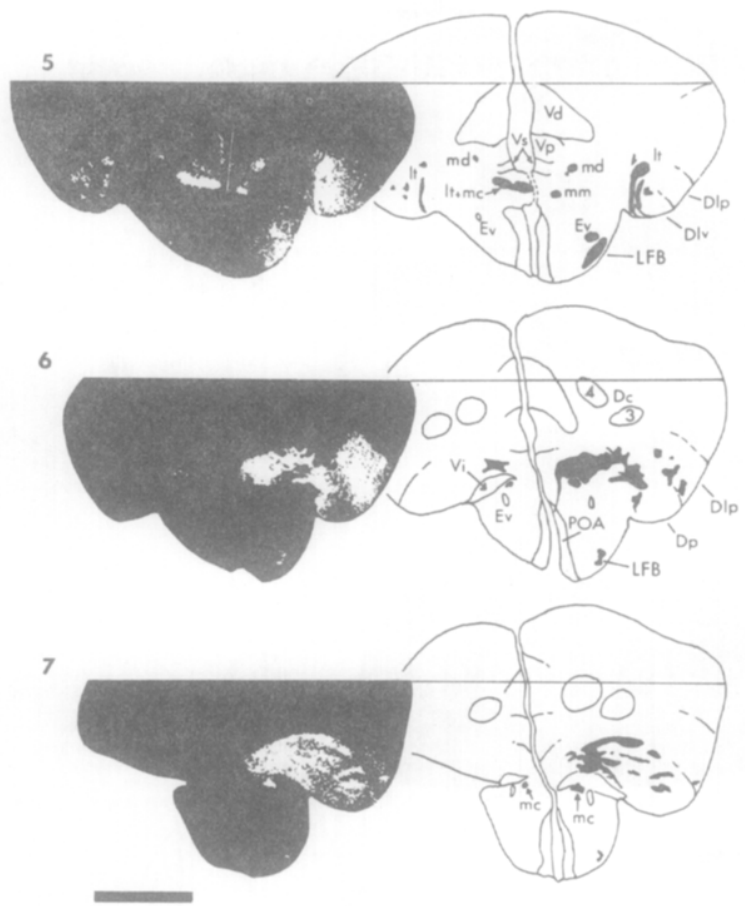

Fig. 6. Additional sections of the brain described in Fig. 5. The dashed line in the midline of section 5 denotes that the tissue was torn.

\section{B. Contralateral Olfactory Projections}

Olfactory bulb fibers cross to the contralateral hemisphere in the interbulbar commissure of Goldstein, anterior commissure, and, presumably, the habenular commissure. The commissure of Goldstein loops over the preoptic recess (Figs. 5.4 and 8A) and ascends to the caudal margin of the contralateral bulb, where the trace became illegible. Fibers also cross dorsally in the anterior commissure to the posterior central and lateral zone of the contralateral hemisphere (Figs. 6.5, 7A, and 9.5). Sparse terminal fields occur in the borders of Vs and Vp and in Vi, Dp, Dlp, and Nt(Fig. 9.5-9.7). The labeled habenular commissure fibers disappeared part way through the commissure, and their destination could not be determined.

\section{Distribution of Labeled Fibers of the Lateral Forebrain Bundle}

Radiolabeling of brain cells in the rostral zone of the hemisphere, ipsilateral to the experimental bulb, revealed three pathways corresponding to parts of the lateral forebrain bundle (Sheldon, 1912; Nieuwenhuys, 1959). Most of the fibers seem to originate rostromedially, in the region 


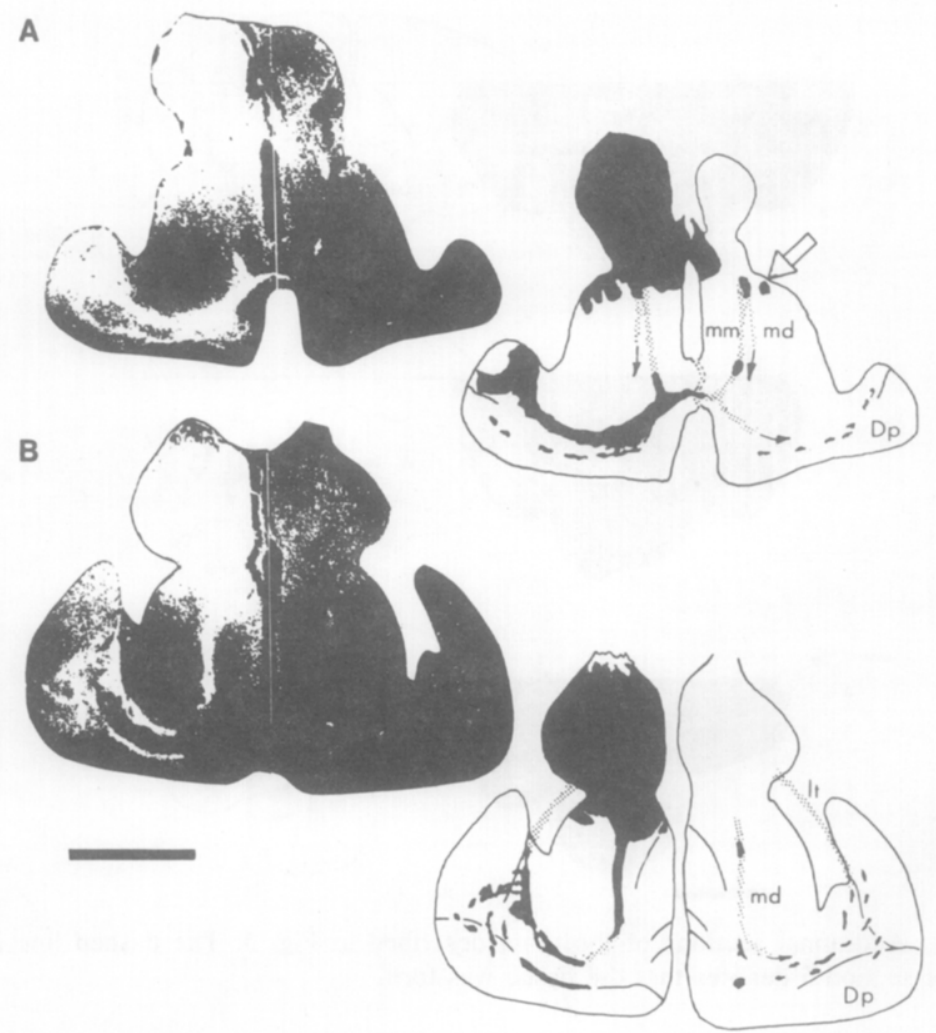

FIg. 7. Autoradiograms of horizontal sections of the telencephalon through (A) and dorsal to (B) the anterior commissure. The zone of diffusion invaded the rostral zone of the hemisphere and the contralateral bulb. Scale bar $=0.5 \mathrm{~mm}$.

of Dm-1 and 2 (Figs. 5.2-3), rather than from Dd or Dlv. The fibers descend to the anterior lateral wall of area $\mathrm{V}$, forming into several illdefined fascicles (Fig. 8A).

One bundle descends in the lateral wall of $\mathrm{V}$ (Figs. 5.4 and 6.5-6.7) to the posterior ventral diencephalon (Fig. 10). The fibers spread out upon entering the diencephalon, but the core of the bundle appears to terminate in the neuropil adjacent to the posterior (NLTp; not illustrated) and inferior (NLTi) parts of nucleus lateral tuberis and the nucleus of the posterior recess (NRP). Another bundle projects caudomedially in $\mathrm{V}$ to the medial aspect of Ev (Figs. 6.5 and 8A). The third, and smallest, bundle courses from the rostral extreme of the lateral wall of $\mathrm{V}$ across the anterior commissure to the corresponding zone of the contralateral area $\mathrm{V}$ (Figs. 5.2-3 and 8A). Its looping path follows that of the commissure of Goldstein. The bundle subsequently turns dorsally then medially to a diffuse terminal field in the region of Dm-1 and 2. 


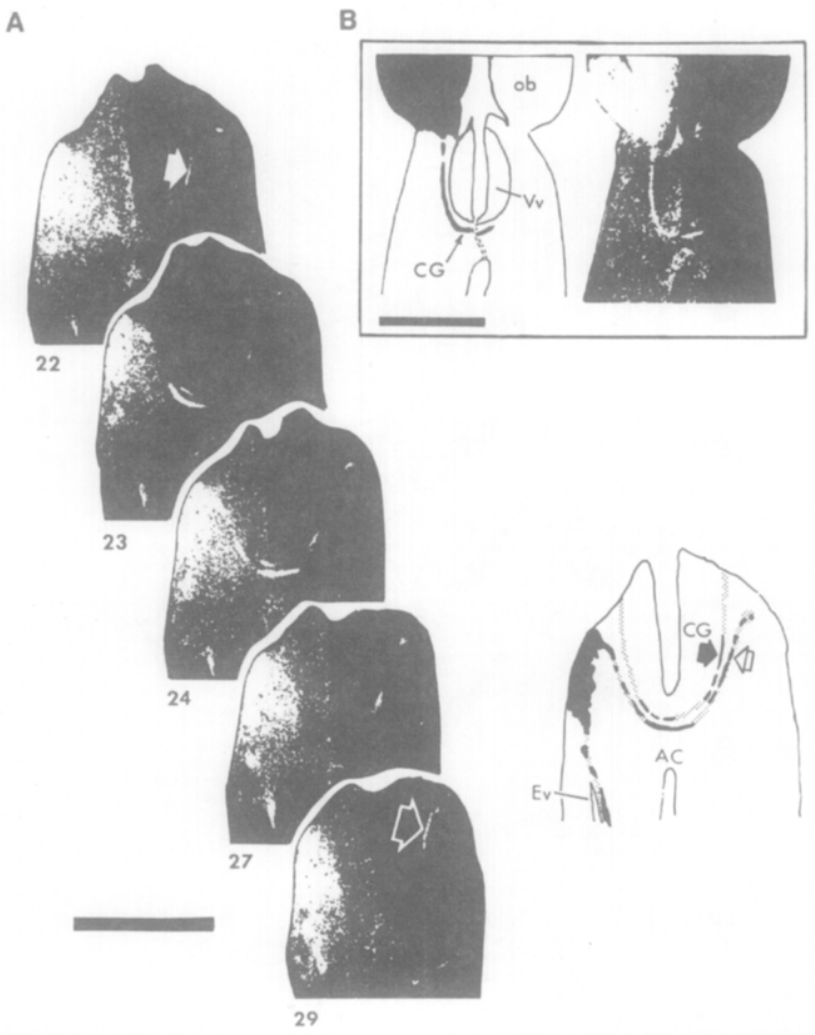

Fig. 8. (A) Autoradiograms of horizontal sections through ventral area V. The experimental bulb and the rostral pole of the ipsilateral hemisphere were intensively labeled (left side of the sections). The contralateral bulb was ablated. Section 22 is the most ventral. The data are summarized in the composite drawing on the right. (B) Horizontal section through area $\mathrm{V}$ in a brain in which the zone of diffusion was largely restricted to the experimental bulb. The contralateral bulb was not ablated. The rostral pole of the ipsilateral hemisphere was only lightly labeled and, correspondingly, there was no evidence of labeled lateral forebrain bundle fibers as seen in (A). Scale bars $=0.5 \mathrm{~mm}$.

\section{DISCUSSION}

\section{Central Projections of the Olfactory Bulb}

\section{A. Hemispheric Targets}

The location of the major afferent olfactory areas of the hemisphere Macropodus, a representative acanthopterygian, resemble those reported for the five ostariophysians that have been examined experimentally. The previous studies employed degeneration methods-Gymnothorax (Scalia \& Ebbesson, 1971), Cyprinus (Ito, 1973), Carassius (Ichikawa, 


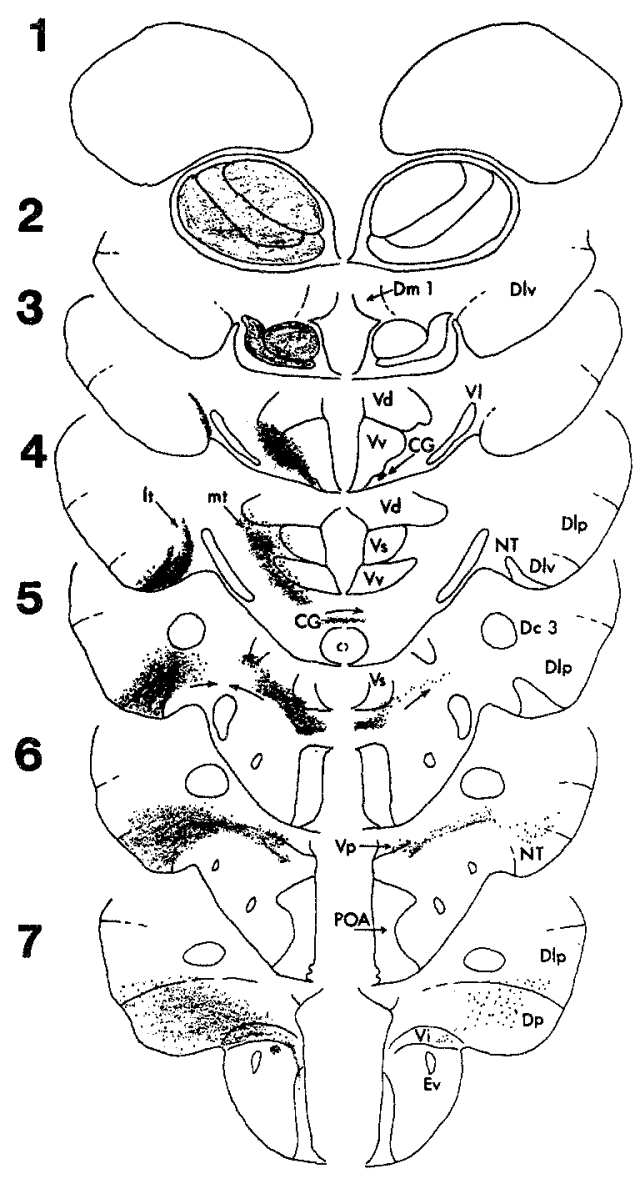

Fig. 9. Summary of the distribution of experimentally localized olfactory tract fibers in the telencephalic hemispheres. The transverse sections were drawn from the reference sections in Fig. 2.

1975; Oka, 1980), and Ictalurus nebulosus (Finger, 1975)—and autoradiography as well-I. punctatus (Bass, 1979). The conclusion that the secondary fibers terminate predominantly in the medial, lateral, and posterior central zones of the hemisphere is generally upheld by our results. While the similarity implies that the topography of the afferent connections is relatively invariant, additional investigations are needed to identify more clearly the brain areas that receive input. Accurate comparisons will require more detailed analysis of the cytoarchitecture of the hemisphere of the different species. The boundaries of the nuclei are in many places very ill-defined. The lateral and posterior-central zones are among the most complex, and their divisions are subject to varying interpretation. The results obtained in six teleosts are briefly compared below. 


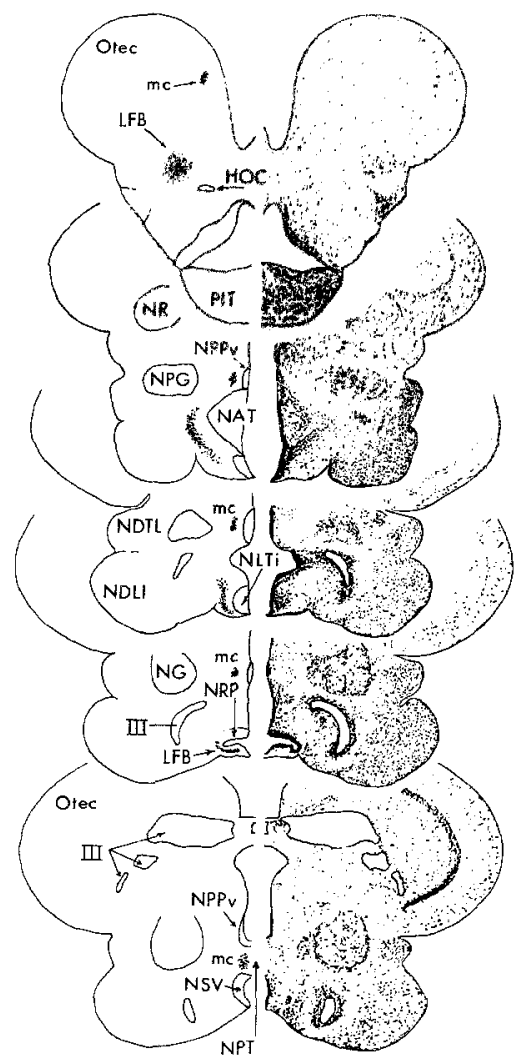

Fig. 10. Summary of the disribution of experimentally localized olfactory tract and lateral forebrain bundle fibers in the diencephalon. The right half of each transverse section is a photomicrograph. These sections and those in Fig. 2 are of the same reference brain.

Area $V$. The distribution of terminal fields in area $\mathrm{V}$ is extensive in some species and relatively restricted in others. The reports for Gymnothorax, I. nebulosus, and Carassius indicate that area $\mathrm{V}$ input is confined to the precommissural region, containing $\mathrm{Vv}$ and the rostral part of Vd, forming a relatively circumscribed "medial" field. In Cyprinus, Ito (1973) localized fiber terminations chiefly in Vl but also in Vd and, we surmise from his illustrations, in $\mathrm{Vv}$ as well. The prominent input to $\mathrm{Vl}$ is interesting in that a similar projection apparently does not occur in the other species. In I. punctatus and Macropodus, nearly all nuclei in $\mathrm{V}$ receive olfactory afferents including $\mathrm{Vi}$, which is topographically contiguous with the "lateral" field.

Area $D$. Olfactory input to $\mathrm{D}$ is restricted to the lateral and the posterior central zones. The lateral zone appears to include the rostral part of Dp in Gymnothorax; of NT and parts of Dp and Dlp in I. nebulosus; and 
of the extreme caudal end of Dlv, the ventral part of Dlp, and all of NT and Dp in I. punctatus. The targets in Macropodus compare closely with those of $I$. punctatus. In Cyprinus, terminals were located in the region of Dlv-Dlp.

The posterior central field includes the posterior part of Dc in Gymnothorax, I. nebulosus, and Carassius. In I. punctatus, fiber terminals are located in the area ventral and lateral to Dc-3. A similar though less elaborated field occurs in Macropodus.

\section{B. Interbulbar Connections}

Connections between the two bulbs have been demonstrated in Gymnothorax and Ictalurus and Macropodus but not Cyprinus nor Carassius. Two projections occur in Ictalurus, in the commissure of Goldstein and a component of the olfactory tracts that cross more dorsally in the anterior commissure. Only the former was detected in Macropodus and only the latter in Gymnothorax. In I. punctatus, Bass (1979) traced interbulbar fibers into the internal and mitral cell layers of the contralateral bulb.

\section{Diencephalic Connections}

The nucleus posterior tuberis receives olfactory input in Ictalurus (Finger, 1975; Bass, 1979) and Macropodus. A similar diencephalic projection was not reported for Gymnothorax, Cyprinus, nor Carassius.

Nieuwenhuys (1967) noted that olfactory fibers project to the habenula. Fibers from the nearby habenular commissure pathway could innervate the nucleus. Some Macropodus autoradiograms suggested the existence of terminals in the core of the nucleus, but the pattern of labeling was unconvincing. Finger (1975) and Bass (1979) traced lateral tract fibers through the habenular commissure to the lateral terminal field of the opposite hemisphere, but they reported no evidence of terminals in the habenula nucleus.

\section{Bilateral Connections}

Among the 6 species, there is marked variation in the number of zones that are innervated bilaterally. In Ictalurus (Finger, 1975; Bass, 1979) and, apparently, Gymnothorax (Scalia \& Ebbesson, 1971), the projections are completely bilateral. Although the bulb innervates the same zones on both sides of the brain, the ipsilateral targets appear to receive more fibers than the contralateral. In Macropodus, bilateral input is restricted to three area $\mathrm{V}$ and three area $\mathrm{D}$ nuclei (Table 2), and the contralateral input is very sparse. The data for Cyprinus indicate that the contralateral input is limited to the ventral zone of V including Vl. In Carassius, only the posterior-central zone of $\mathrm{D}$ is innervated bilaterally (Ichikawa, 1973; Oka, 1980). 


\section{Lateral Forebrain Bundle}

Experimental studies of the efferents of the rostral zone of area D have apparently not been previously reported. The three projections detected in Macropodus compose only a small part of the lateral forebrain bundle, which is the largest tract system in the teleost forebrain (Johnston, 1911; Sheldon, 1912; Nieuwenhuys, 1959). The system includes many bundles connecting the diencephalon and virtually all parts of area D. Some bundles decussate in the anterior commissure as described, for example, in Cyprinus (Sheldon, 1912) and Gasterosteus (Nieuwenhuys, 1959). In Gasterosteus, Nieuwenhuys observed that fibers arising from $\mathrm{Dm}$ seem to project to $\mathrm{Dm}$ in the opposite hemisphere, forming a commissure. The commissural projection detected in Macropodus clearly fits this description.

The ventral entopenduncular nucleus, which is embedded in the lateral forebrain bundle (Nieuwenhuys, 1959), is apparently innervated by cells of rostral area D in Macropodus. Unfortunately, the identity of the cells is unclear in our limited material. The cells-of-origin of the diencephalic projection also remains to be clarified. Its distribution in the ventral diencephalon partly resembles that of the so-called strio-lobar bundle described in Eugerres and Holocentras. Vanegas and Ebbesson (1976) traced degenerating telencephalic fibers following unilateral ablation of the entire hemisphere in these perciform fishes. The strio-lobar, however, ends in the inferior lobe in the region of the lateral recess of the III ventricle, and no projection to the nearby posterior recess was indicated. While the projection in Macropodus passes near the lateral recess, it ends medially near the NLTp, NLTi, and NRP. Thus, its relationship to the strio-lobar is unclear.

\section{Correlations with Previous Neural and Behavioral Findings in Macropodus}

Hemispheric and bulbar lesions impair reproductive functions in $M a$ cropodus. The lesions could act by destroying brain areas that potentiate reproduction or by disrupting essential input to such areas. Neurons that regulate reproductive processes are thought to include those which concentrate sex steroids (Morrell, Kelley, \& Pfaff, 1975). In the male $M a$ cropodus, steroid-concentrating cells occur in $\mathrm{Vv}$, the ventral zone of POA, NLT, the nucleus of the lateral recess of the third ventricle, and the extreme caudal extent of NPPv (Davis, Morrell, \& Pfaff, 1977).

\section{A. Afferent Olfactory Connections}

The present experiments imply that steroid-concentrating cells in $\mathrm{Vv}_{\mathrm{v}}$ could receive olfactory innervation. Input to the cells in ventral POA seems less likely, since the olfactory afferents were traced to dorsal 
POA. The caudal branch of the olfactory tract courses very close to NPPv (Fig. 10). Though the fibers did not visibly penetrate among the nucleus, NPPv fibers might extend laterally into the olfactory pathway.

Bilateral bulbectomy results in increased egg cannibalism in the male, but the incidence of various sexual behaviors including spawning is not significantly affected (Davis et al., 1981). Investigations of partial lesions indicated that medial olfactory tract fibers, possibly including those which project to $\mathrm{Vv}$, are necessary for normal egg care. Whether $\mathrm{Vv}$ plays a role in egg care or spawning in Macropodus remains to be examined. However, Kyle and Peter (1979) showed that, in male Carassius, the incidence of spawning is decreased following administration of a localized lesion in Vs-Vv. A similar localized lesion in POA does not impair spawning. Spawning is also decreased following blockade of olfactory input (Partridge, Liley, \& Stacey, 1976). Thus, it is possible that the functions of the olfactory input to the $\mathrm{Vs}-\mathrm{Vv}$ region (Ichikawa, 1975) include potentiation of spawning.

\section{B. Lateral Forebrain Bundle Connections}

Telencephalic lesion experiments in Macropodus have so far shed little light on the behavioral functions of the complex lateral forebrain bundle system. Though male reproductive behaviors are decreased following complete removal of the hemispheres to the level of POA (Kassel \& Davis, 1977), the same behaviors are unaffected by extensive removal of area D and the posterior dorsal parts of V (Davis et al., 1981). If the lateral forebrain connections are located predominantly in area D, as generally believed (Sheldon, 1912; Nieuwenhuys, 1959), they apparently do not play a limiting role in controlling the reproductive behaviors that we observed.

The extensive area $\mathrm{D}$ lesions referred to above selectively spared the extreme rostromedial zone, including Dm-1 and in most cases Dm-2 as well. The link between rostral Dm and the steroid-concentrating areas NLTi and NLTp, detected in the present experiments, could have been spared. The possibility that a Dm-NLT projection is necessary for spawning is dashed, however, by the finding that the behavior is not decreased following bilateral ablation of the rostral end of the hemisphere (Davis et al., 1981).

\section{Evidence of Retrograde Transport of Radiolabeled Substance}

Kunzle (1977) reported that while tritiated proline is particularly effective in labeling anterograde axonal flow (Elam \& Agranoff, 1971), certain neurons in mammal brain show evidence of labeled retrograde flow. Perikarya in specific brain regions contain high concentrations of radioactivity following spinal application of tritiated proline. We detected similarly labeled cells in the hemisphere of Macropodus in a pilot study 
(Davis \& Agranoff, 1977), in which the incorporation period was only $5 \mathrm{hr}$. Some brains in the present experiment, with a 10-day incorporation period, showed labeled perikarya, but they were less abundant or conspicuous.

Though the radiolabeled cells are few and widely scattered in the hemisphere, a distinct cluster occurs in the ipsilateral Nt-Dlp region, as illustrated by Davis and Agranoff (1977). Efferent cells are reported to occur in this region, among others, in the brain of Carassius (Oka, 1980). The coincidence suggests that the radiolabeled cells in Macropodus could be efferents whose terminals take up and transport tritiated proline or metabolite. If such transport occurs, the tracts that we traced must be regarded as containing not only secondary olfactory afferents but an unknown number of efferents as well.

\section{REFERENCES}

Ariens-Kappers, C. U., Huber, G. C., \& Crosby, E. C. (1936). The Comparative Anatomy of the Nervous System of Vertebrates, Including Man. Reprinted 1960. New York: Hafner.

Bass, A. H. (1979). Telencephalic afferents and efferent in the channel catfish, Ictalurus punctatus. Ph.D. Dissertation, Ann Arbor: University of Michigan.

Davis, R. E. (1979). Olfactory bulb neurons terminate in the receptor epithelium. Neurosciences Abstracts, $5,127$.

Davis, R. E., \& Agranoff, B. W. (1977). Microimplantation of $\left[{ }^{3} \mathrm{H}\right]$ proline on a single bead of ion exchange resin. Brain Research, 124, 341-346.

Davis, R. E., Kassel, J., \& Martinez, M. (1981). The telencephalon and reproductive behavior in the teleost, Macropodus opercularis (L.): Effects of lesions on the incidence of spawning and egg cannibalism. In P. R. Laming (Ed.), Neuroethology of Lower Vertebrates, pp. 239-255. London and New York: Cambridge Univ. Press.

Davis, R. E., Kassel, J., \& Schwagmeyer, P. (1976). Telencephalic lesions and behavior in the teleost, Macropodus opercularis: Reproduction, startle reaction, and operant behavior in the male. Behavioral Biology, 18, 165-177.

Davis, R. E., Morrell, J. J., \& Pfaff, D. W. (1977). Autoradiographic localization of sex steriod concentrating cells in the brain of the teleost Macropodus opercularis (Osteichthyes:Belontiidae). General and Comparative Endocrinology, 33, 496-505.

Davis, R. E., Reynolds, R. C., \& Ricks, A. (1978). Suppression behavior increased by telencephalic lesions in the teleost, Macropodus opercularis. Behavioral Biology, 24, $32-48$.

Demski, L. S., Bauer, D. H., \& Gerald, J. W. (1975). Sperm release evoked by electrical stimulation of the fish brain: A functional-anatomical study. Journal of Experimental Zoology, 191, 215-232.

Demski, L. S., \& Knigge, K. M. (1971). The telencephalon and hypothalamus of the bluegill (Lepomis macrochirus): Evoked feeding, aggressive and reproductive behavior with representative frontal sections. Journal of Comparative Neurology, 143, 1-16.

Elam, J. S., \& Agranoff, B. W. (1971). Rapid transport of protein in optic system of the goldfish. Journal of Neurochemistry, 18, 375-387.

Finger, T. (1975). The distribution of the olfactory tracts in the bullhead, Ictalurus nebulosus. Journal of Comparative Neurology, 161, 125-142.

Flood, N. B., Overmier, J. B., \& Savage, G. E. (1976). Teleost telencephalon and Learning: An interpretive review of data and hypotheses. Physiology and Behavior, 16, 783-798. 
Holmgren, N. (1920). Zur Anatomie und Histologie des Vorder- und Zwischenhirns der Knochenfische. Acta Zoologica, 1, 137-315.

Holmgren, N. (1922). Points of view concerning forebrain morphology in lower vertebrates. Journal of Comparative Neurology, 34, 391-459.

Ichikawa, M. (1975). The central projections of the olfactory tract in the goldfish, Carassius auratus. Journal of the Faculty of Science, Tokyo, 13(3), 257-263.

Ito, H. (1973). Normal and experimental studies on synaptic patterns in the carp telencephalon, with special reference to the secondary olfactory termination. Journal Hirnforschung, 14, 237-245.

Johnston, J. B. (1911). The telencephalon of ganoids and teleosts. Journal of Comparative Neurology, 21, 489-591.

Kassel, J., \& Davis, R. E. (1977). Recovery of function following simultaneous and serial telencephalon ablation in the teleost, Macropodus opercularis, Behavioral Biology, 21, 489-499.

Kassel, J., Davis, R. E., \& Schwagmeyer, P. (1976). Telencephalic lesions and behavior in the teleost, Macropodus opercularis: Further analysis of reproductive and operant behavior in the male. Behavioral Biology, 18, 179-188.

Kopriwa, B. M., \& Leblond, C. P. (1962). Improvements in the coating technique for radioautography. Journal of Histochemistry and Cytochemistry, 10, 269-284.

Kunzle, H. (1977). Evidence for selective axon-terminal uptake and retrograde transport of label in cortico- and rubrospinal systems after injection of ${ }^{3} \mathrm{H}$-proline. Experimental Brain Research, 28, 125-132.

Kule, A. L., \& Peter, R. E. (1979). Effect of brain lesions on spawning behaviour in the male goldfish. Presented at the Canada West Society for Reproductive Biology, University of Saskatchewan, Canada.

Laming, P. R. (1980). Electroencephalographic studies on arousal in the goldfish (Carassius auratus). Journal of Comparative Physiological Psychology, 94 (2), 238-254.

Laming, P. R. (1981). The physiological basis of alert behaviour in fish. In P. R. Laming (Ed.) Neuroethology of Lower Vertebrates, pp. 203-222. Cambridge: Cambridge University Press.

Landreth, G. E., \& Agranoff, B. W. (1976). Explant culture of adult retina: effect of prior optic nerve crush. Brain Research, 118, 299-303.

Morrell, J. I., Kelley, D. B., \& Pfaff, D. W. (1975). Sex steroid binding in the brains of vertebrates. In K. M. Knigge and D. E. Scott (Eds.), Brain-Endocrine Interaction II: The Ventricular System in Neuroendocrine Mechanisms, pp. 230-256. White Plains, N.Y.: Phiebig.

Nieuwenhuys, R. (1959). The structure of the telencephalon of the teleost Gasterosteus aculeatus. Netherlands Central Institute for Brain Research, Proceedings, Series $C$, 62(4), 341-362.

Nieuwenhuys, R. (1962). Trends in the evolution of the actinopterygian forebrain. Journal of Morphology, 111, 69-88.

Nieuwenhuys, R. (1963). The comparative anatomy of the actinopterygian forebrain. Journal fur Hirnforschung, 6(3), 1971-192.

Nieuwenhuys, R. (1967). Comparative anatomy of olfactory centres and tracts. Progress in Brain Research, 23, 1-64.

Northcutt, R. G., \& Braford, M. R. (1980). New observations on the organization and evolution of the telencephalon of actinopterygian fishes. In S. O. E. Ebbesson (Ed.), Comparative Neurology of the Telencephalon, pp. 41-98, New York.

Oka, Y. (1980). The origin of centrifugal fibers to the olfactory bulb in the goldfish, Carassius auratus: An experimental study using the fluorescent dye primuline as a retrograde tracer. Brain Research, 185, 215-225.

Partridge, B. L., Liley, N. R., \& Stacey, N. E. (1976). The role of pheromones in the sexual behavior of the goldfish. Animal Behaviour, 24, 291-299. 
Peter, R., \& Gill, V. (1975). Stereotoxic atlas and technique for forebrain nuclei of goldfish. Carassius auratus. Journal of Comparative Neurology, 159, 69-101.

Scalia, F., \& Ebbesson, S. O. E. (1971). The central projections of the olfactory bulb in a teleost (Gymnothorax funebris). Brain, Behavior and Evolution,4, 376-399.

Schwagmeyer, R., Davis, R. E., \& Kassel, J. (1977). Telencephalic lesions and behavior in the teleost, Macropodus opercularis (L.): Effects of telencephalon and olfactory bulb ablation on spawning and foamnest building. Behavioral Biology, 20, 463-470.

Sheldon, R. E. (1912). The olfactory tracts and centers in teleosts. Journal of Comparative Neurology, 22, 177-339.

Vanegas, H., \& Ebbesson, S. O. E. (1976). Telencephalic projections in two teleost species. Journal of Comparative Neurology 165(2), 181-195.

Wiitanen, J. T. (1969). Selective silver impregnation of degenerating axons and axon terminals in the central nervous system of the monkey (Macaca mulatta). Brain Research, 14, 546-548. 